\title{
Muséologies
}

Les cahiers d'études supérieures

muséologies

\section{Les musées en région au Québec : essai de définition}

\section{Meggie Savard}

Volume 6, numéro 1, 2012

URI : https://id.erudit.org/iderudit/1011535ar

DOI : https://doi.org/10.7202/1011535ar

Aller au sommaire du numéro

\section{Éditeur(s)}

Association Québécoise de Promotion des Recherches Étudiantes en Muséologie (AQPREM)

ISSN

1718-5181 (imprimé)

1929-7815 (numérique)

Découvrir la revue

Citer cet article

Savard, M. (2012). Les musées en région au Québec : essai de définition. Muséologies, 6(1), 119-135. https://doi.org/10.7202/1011535ar

\section{Résumé de l'article}

La réalité des musées québécois éloignés des grandes agglomérations urbaines diffère substantiellement de celle des institutions évoluant en milieu métropolitain. Meggie Savard définit l'environnement dans lequel évoluent ces institutions en évoquant une certaine forme d'autonomie culturelle, la présence d'une forte identité régionale et leur composition démographique (à prévalence caucasienne, homogène et francophone), le phénomène de l'exode rural, un faible niveau de scolarisation des habitants ainsi qu'un taux de chômage supérieur à la moyenne nationale. Malgré les difficultés avec lesquelles ces musées doivent composer, les divers paliers de gouvernance local, provincial ou national ne leur accordent aucune reconnaissance ni statut particulier. En s'appuyant sur ce constat, Meggie Savard tente de définir et de circonscrire le " musée régional » en l'opposant au musée urbain par le biais d'une revue d'écrits et l'étude d'un cas typique observé au Saguenay-Lac-Saint-Jean afin de proposer quelques idées d'appui financier adaptées aux besoins propres à ces institutions.
Tous droits réservés (C) Association Québécoise de Promotion des Recherches Étudiantes en Muséologie (AQPREM), 2012
Ce document est protégé par la loi sur le droit d'auteur. L’utilisation des services d'Érudit (y compris la reproduction) est assujettie à sa politique d'utilisation que vous pouvez consulter en ligne.

https://apropos.erudit.org/fr/usagers/politique-dutilisation/ 
Article cinq

Les musées en région au Québec:

essai de définition

Meggie Savard 
La réalité des musées québécois éloignés des grandes agglomérations urbaines diffère substantiellement de celle des institutions évoluant en milieu métropolitain. Meggie Savard définit l'environnement dans lequel évoluent ces institutions en évoquant une certaine forme d'autonomie culturelle, la présence d'une forte identité régionale et leur composition démographique (à prévalence caucasienne, homogène et francophone), le phénomène de l'exode rural, un faible niveau de scolarisation des habitants ainsi qu'un taux de chômage supérieur à la moyenne nationale. Malgré les difficultés avec lesquelles ces musées doivent composer, les divers paliers de gouvernance local, provincial ou national ne leur accordent aucune reconnaissance ni statut particulier. En s'appuyant sur ce constat, Meggie Savard tente de définir et de circonscrire le «musée régional" en l'opposant au musée urbain par le biais d'une revue d'écrits et l'étude d'un cas typique observé au SaguenayLac-Saint-Jean afin de proposer quelques idées d'appui financier adaptées aux besoins propres à ces institutions.

Native du Saguenay-Lac-Saint-Jean, Meggie Savard est titulaire d'un baccalauréat en histoire de l'art de l'Université de Montréal et d'une maîtrise en muséologie (2010) de la même université. Durant ses études universitaires, elle s'est familiarisée avec le milieu muséal en travaillant comme guide-animatrice au sein de divers musées montréalais. Conservatrice du Musée de Charlevoix depuis juin 2010, elle y a notamment réalisé l'exposition à succès Cap sur les bateaux blancs (2011-2012). Meggie Savard a également œuvré comme consultante en muséologie dans la région de Charlevoix; elle a réalisé la nouvelle exposition permanente du Centre d'interprétation du patrimoine de Notre-Dame-des-Monts. Cet article est extrait de son travail dirigé de maîtrise. meg_savard@hotmail.com 
Les musées sont nés des passions de collectionneurs, de leur volonté de protéger et de diffuser la richesse du patrimoine qu'ils chérissent. Nés de projets individuels, ils sont graduellement devenus des centres de recherche faisant figure d'autorité dans leur domaine auprès de la population et participant à l'avancement des connaissances. Gardiens du patrimoine, ils se sont institutionnalisés. Dès la première moitié du XIX ${ }^{\mathrm{e}}$ siècle, les premiers musées en région ${ }^{1}$ au Québec ont fait leur apparition ${ }^{2}$. Les populations locales, dont l'histoire est encore très récente, ressentaient le besoin de se doter d'un outil pour protéger leur patrimoine.

Le sujet des musées en région au Québec a rarement fait l'objet d'études, autres que celles menées par le muséologue Philippe Dubé et celles menées par le gouvernement à des fins statistiques. Situées hors des grands centres urbains, ces institutions représentent aujourd'hui environ $60 \%$ des musées québécois $^{3}$ et sont réparties sur un territoire représentant $98,2 \%$ de la superficie totale da la province $^{4}$. Un constat s'impose : les besoins réels de la majorité des musées de la province demeurent méconnus. Or, dans un contexte de mondialisation et d'uniformisation des cultures, les musées québécois en région jouent un

\section{L'expression "musée en région " sera utilisée dans} cette recherche, mettant ainsi l'expression "musée régional " de côté. La différence entre ces deux concepts sera expliquée plus loin.

2 FALLU, Jean-Marie. «L'éveil muséal des régions du Québec ", Musées (Montréal), vol. 28, 2009, p. 87. L'auteur se base sur des données de l'Observatoire de la culture et des communications du Québec publiées en 2009: $<$ www.stat.gouv.qc.ca/donstat/societe/culture_comnc/ musees>. Des 310 institutions membres de la Société des musées québécois, 185 se trouvent en région.

3 Id., p. 87. L'auteur se base sur des données de l'Observatoire de la culture et des communications du Québec publiées en 2008: <www.stat.gouv.qc.ca/donstat/societe/ culture_comnc/musees $>$.

4 INSTITUT DE LA STATISTIQUE DU QUÉBEC. Profil des régions. <http://www.stat.gouv.qc.ca/regions/profils/ region_03/region_03_00.htm> (consulté le 29 janvier 2009).

5 Le gouvernement provincial utilise l'expression

«institution muséale " pour désigner les institutions suivantes: le centre d'interprétation, le lieu historique, le musée et le centre d'exposition. Pour le MCCCf, la catégorie «musée " comprend les musées proprement dits ainsi que les écomusées, selon la définition qu'en donne la Direction des musées de France. Le Ministère reconnaît que «Le musée [à la différence rôle plus qu'essentiel dans la sauvegarde de notre patrimoine et dans la définition de notre identité.

En effet, ces institutions muséales ${ }^{5}$ régionales évoluent en même temps que la société qu'elles servent et sont par conséquent au diapason de ses problèmes. Dispersées sur un grand territoire à la densité de population très faible, elles fonctionnent avec peu de moyens financiers. N'étant pas admissibles à un support financier particulier provenant du gouvernement pour contrebalancer cet état de fait, ces institutions doivent tout de même répondre aux mêmes exigences que les musées des grands centres urbains $^{6}$.

Au Québec, la régionalisation passe du stade de l'État décentralisateur au stade de l'État accompagnateur ${ }^{7}$. Toutefois, même si le gouvernement demande aux régions d'être plus autonomes dans leur développement, les ressources financières et humaines manquent ${ }^{8}$ et peuvent pousser notamment les musées à mettre de côté leurs rôles premiers ${ }^{9}$ pour pouvoir simplement éviter un trop grand déficit. Ces institutions sont présentement en crise de fonctionnement, puisqu'elles ne sont plus en mesure de bien remplir leurs rôles ${ }^{10}$.

des centres d'interprétation et d'exposition de même des lieux historiques] accomplit l'ensemble des fonctions muséales: acquisition, conservation, recherche, éducation, action culturelle et diffusion. " (MINISTÈRE DE LA CULTURE, DES COMMUNICATIONS ET DE LA CONDITION FÉMININE, Politique muséale. Vivre autrement... la ligne du temps. Québec. <http://www.mcccf.gouv.qc.ca/publications/ politiquemuseale.pdfa> [consulté le 29 janvier 2010].) 6 Critères relevant du code de déontologie de l'ICOM. (INTERNATIONAL COUNCIL OF MUSEUMS. Évolution de la définition du musée selon les statuts de l'ICOM (2007-1946).<http://icom.museum/hist_def_fr.html> [consulté le 28 janvier 2009].)

7 MORIN, Richard. La régionalisation au Québec.

Les mécanismes de développement et de gestion des territoires régionaux et locaux, 1960-2000. Montréal: Éditions SaintMartin, 2006.

8 Id.

9 Ces rôles premiers et fondamentaux peuvent être résumés par la conservation et la protection du patrimoine de même que par la diffusion et la vulgarisation de savoirs scientifiques. 10 DUBÉ, Philippe. "À propos d'une certaine muséologie en région ". Ethnologies, vol. 24, n 2, 2002. <http://www. erudit.org/revue/ethno/2002/v24/n2/006644ar.html> (consulté le 2 janvier 2010). 
Cet article $^{11}$ ne se veut pas une démonstration du sous-financement du secteur culturel en région, puisqu'un mémoire sur le sujet a déjà été déposé à la Commission parlementaire sur la culture ${ }^{12}$. L'objectif est plutôt de définir les musées situés sur ces territoires afin de tendre vers la reconnaissance d'un autre type d'institution muséale: le musée en région. Reconnaître leur spécificité et par le fait même leurs besoins constitue la première étape d'une démarche visant à la reconnaissance des problèmes et des enjeux qui leur sont spécifiques dans l'espoir d'améliorer leur situation. D'après l'information diffusée sur le site officiel du ministère de la Culture, des Communications et de la Condition féminine (MCCCf), cette typologie a été développée aux fins des programmes provinciaux d'aide financière visant le secteur muséal.

La définition du musée en région s'articulera en quatre étapes: 1) la caractérisation de l'institution par sa situation géographique; 2) l'interprétation de l'institution par ses caractéristiques intrinsèques; 3) l'analyse des données récoltées sur le terrain; 4) la synthèse des résultats dans le but de répondre à une simple question: "Qu'est-ce qu'un musée en région au Québec?». L'ensemble de cette démarche combine à la fois une revue des écrits sur des sujets ciblés et des entretiens avec des professionnels du milieu.

\section{Méthodologie}

À une revue de la littérature s'ajoutera une analyse dont le terrain d'étude est la région du Saguenay-Lac-Saint-Jean et les musées

11 Je tiens à remercie Lucie Couillard, muséologue, de ses précieux conseils lors de la rédaction de cet article. 12 THOMIN, Jean-Paul et Suzanne CHASSÉ. Le soutien des institutions régionales au Québec: un levier essentiel pour la démocratisation de la culture. Mémoire présenté à la Commission parlementaire sur la culture du Québec, novembre 1991. 13 L'approche localisée est une des deux approches ethnologiques du terrain et est décrite brièvement par l'ethnologue Jocelyne MATHIEU: «La région: un terrain ou un concept? Approche ethnologique ». In. HARVEY, Fernand (dir.). La région culturelle. Problématique interdisciplinaire. Québec: Institut québécois de recherche sur la culture, 1994, p. 97-110. qui se trouvent sur ce territoire. Cette recherche s'appuie sur une approche localisée du sujet, c'est-à-dire que le territoire est étudié dans le but de comprendre localement un phénomène afin d'en arriver à une compréhension globale par la généralisation de certaines conclusions ${ }^{13}$. Les conclusions de cette analyse pourraient donc être reportées sur d'autres régions québécoises de même type ${ }^{14}$.

Le choix du Saguenay-Lac-Saint-Jean comme terrain se justifie à plusieurs niveaux. Tout d'abord, cette région correspond à une région de type excentrique, ce qui facilite l'identification de phénomènes. Selon la typologie d'Andrée Fortin et Fernand Harvey ${ }^{15}$ (tableau 1), ce type de territoire (appelé "périphérique" par les auteurs) est caractérisé par une certaine autonomie culturelle qu'on observe plus difficilement dans les autres types de régions et qui est favorisée par un éloignement important des grands centres. Toujours selon ces sociologues, ces territoires sont les moins influencés par les régions centrales et tous les services et produits culturels qu'elles offrent. Comme le déclare Chad Gaffield, les chercheurs du Centre interuniversitaire de recherche sur les populations (SOREP) confirment également la validité du Saguenay-Lac-Saint-Jean comme terrain d'étude du phénomène régional: «les chercheurs [du SOREP] n'ont pas seulement démontré que l'espace du Saguenay-LacSaint-Jean constituait un laboratoire valable mais ils ont également confirmé le fait que ce territoire était une 'région' à la fois liée et distincte par rapport aux espaces limitrophes ${ }^{16}$.

14 La typologie des territoires régionaux québécois est abordée plus loin dans cet article.

15 HARVEY, Fernand et Andrée FORTIN. "Production et diffusion culturelles en région : émergence d'une nouvelle dynamique». In. HARVEY, Fernand et Andrée FORTIN (dir.). La nouvelle culture régionale. Québec: Institut québécois de recherche sur la culture, 1995, p. 13-34. 16 GAFFIELD, Chad. "La région: une combinaison spécifique d'éléments non spécifiques ». In. HARVEY, La région culturelle. Problématique interdisciplinaire, op. cit., p. 29. 
Tableau 1

Essai de typologie des espaces culturels régionaux au Québec et au Canada français

\begin{tabular}{|ll|l|}
\hline 1. & \multicolumn{2}{l|}{ Régions centrales } \\
& (concentration de la création/production/diffusion/et ou l'appareil d'État) \\
\hline 1.1 & La métropole culturelle: & Montréal et sa banlieue \\
\hline 1.2 & Les capitale culturelles: & Québec et Ottawa et leurs banlieues \\
\hline
\end{tabular}

\begin{tabular}{|ll|l|}
\hline 2. & $\begin{array}{l}\text { Régions satellites } \\
\text { (à proximité des grands centres et sans infrastructures culturelles complètes) }\end{array}$ \\
\hline 2.1 & Régions satellites de Montréal: & $\begin{array}{l}\text { Montérégie, Laval, Laurentides, } \\
\text { Lanaudière }\end{array}$ \\
\hline 2.2 & Régions satellites de Québec: & $\begin{array}{l}\text { Lévis-Lobtinière, Côte-du-Sud, Beauce, } \\
\text { Amiante, Côte-de-Beaupré, Charlevoix, } \\
\text { Portneuf }\end{array}$ \\
\hline
\end{tabular}

\begin{tabular}{|c|c|c|}
\hline 3. & \multicolumn{2}{|c|}{$\begin{array}{l}\text { Régions intermédiaires } \\
\text { (situées entre deux pôles urbains d'importance et disposant de ressources } \\
\text { et d'infrastructures variées) }\end{array}$} \\
\hline 3.1 & Entre Montréal et Québec: & $\begin{array}{l}\text { Mauricie, Centre du Québec, Bois-Francs, } \\
\text { Estrie, Outaouais }\end{array}$ \\
\hline 3.2 & Entre Montréal et Ottawa: & Outaouais \\
\hline
\end{tabular}

\begin{tabular}{|ll|l|}
\hline 4. & $\begin{array}{l}\text { Régions périphériques } \\
\text { (excentriques aux grands centres et disposant de ressources et d'infrastructures } \\
\text { variées) }\end{array}$ & \multicolumn{2}{l|}{} \\
\hline 4.1 & Régions du Québec oriental: & $\begin{array}{l}\text { Saguenay-Lac-St-Jean, Côte-Nord, } \\
\text { Bas-St-Laurent, Gaspésie, } \\
\text { Îles-de-la-Madeleine }\end{array}$ \\
\hline 4.2 & $\begin{array}{l}\text { Régions du Québec occidental } \\
\text { ou nordique: }\end{array}$ & $\begin{array}{l}\text { Abitibi-Témiscamingue, Baie-James, } \\
\text { Nunavik }\end{array}$ \\
\hline
\end{tabular}

\section{Régions du Canada français \\ (liens culturels variables avec le Québec)}

5.1 Régions à forte densité francophone:

5.2 Régions à faible densité francophone:
Nouveau-Brunswick: Madawaska, Nord-Est, Moncton

Ontario: Nord-Est, Prescon-Russel

îlots francophones dans les Maritimes, le Sud de l'Ontatio et l'Ouest canadien

Source: HARVEY et FORTIN, La nouvelle culture régionale 


\section{Le musée en région et le musée régional}

Dans un premier temps, il importe de préciser que la notion de "musée régional " doit être différenciée de celle de "musée en région ".

En effet, ces deux expressions réfèrent à deux sous-catégories d'institutions muséales régionales. Au terme de quelques entretiens avec des professionnels du milieu muséal au SaguenayLac-Saint-Jean ${ }^{17}$, les définitions suivantes ont pu être dégagées:

- Musée régional: L’appellation «musée régional ", au Saguenay-Lac-Saint-Jean, réfère à l'institution qui a pour mission la protection et la diffusion du patrimoine régional et qui possède donc une collection qui représente l'ensemble des communautés du territoire administratif. De plus, cette institution de plus grande dimension assure un leadership en matière de muséologie et doit aider dans la mesure du possible les autres musées de la région à se développer.

- Musée en région: L'appellation "musée en région ", pour les professionnels du simplement à un musée situé dans la région administrative du Saguenay-Lac-Saint-Jean autre que le Musée régional.

\section{La caractérisation de l'institution par sa situation géographique}

Qu'est-ce qu'un musée en région au Québec? En tentant de répondre à cette question, nous relions d'abord l'institution à sa situation géographique: «un musée en région au Québec est simplement un musée situé en région ». Nous aurions donc réussi à définir rapidement ce concept. Or, une question essentielle s'impose: qu'est-ce qu'une région au Québec? Plusieurs auteurs provenant de différentes disciplines ont tenté de le déterminer sans toutefois y parvenir. À cette grande question sont donc associées différentes réponses, toutes partielles.

17 Entretiens avec les directeurs des musées ainsi qu'avec un membre de l'équipe de la Direction régionale du MCCCf, réalisés les 19, 20, 21 et 22 janvier 2010.

18 MORIN, La régionalisation au Québec..., op. cit.

Il importe ici de tenter d'en dégager une qui soit adaptée aux objectifs de cette recherche, dont la problématique est liée à la définition d'un musée en région et à la reconnaissance d'un nouveau type d'institution muséale, s'ily a lieu.

\section{Du point de vue de l'urbaniste}

Dans son ouvrage La régionalisation au Québec. Les mécanismes de développement et de gestion des territoires régionaux et locaux ${ }^{18}$, Richard Morin aborde les trois grandes phases de régionalisation débutant après la Deuxième Guerre mondiale et en explore les bienfaits et les méfaits sur le développement régional.

Les régions administratives au Québec ont été créées en partie lors de la première phase de décentralisation en 1966 et 1967 par le gouvernement québécois. Au nombre de dix, leur utilité était, «d'une part, de rationaliser les multiples découpages régionaux déjà existants et, d'autre part, de se munir d'un cadre de référence pour planifier le développement de l'ensemble de son territoire " ${ }^{19}$. Durant les décennies 1980 et 1990, la deuxième phase de régionalisation a fait naître notamment six autres régions administratives ${ }^{20}$ (ill. 1). La troisième phase de régionalisation a débuté en 2003 et se poursuit encore aujourd'hui. L'État tend maintenant vers « une diminution de ses dépenses ainsi qu'un nouveau partage des responsabilités avec les régions et une plus grande responsabilisation des élus locaux ${ }^{21}$. Cette nouvelle phase a comme conséquences une plus grande responsabilisation des élus, mais aussi une baisse de participation de la société civile au développement régional. Selon Morin, cette phase est plus caractérisée par une déconcentration des services qu'une réelle décentralisation des pouvoirs, étant donné que les élus sont choisis par le gouvernement et non par la population. De nouveaux pouvoirs sont ainsi donnés aux municipalités sans que de nouvelles ressources leur soient octroyées.

19 Id., p. 28

20 Conception de la région comme espace fonctionnel polarisé par la ville.

21 MORIN, La régionalisation au Québec..., op. cit., p. 45. 
Illustration 1

\section{Les régions administratives du Québec} et la région du Saguenay-Lac-Saint-Jean

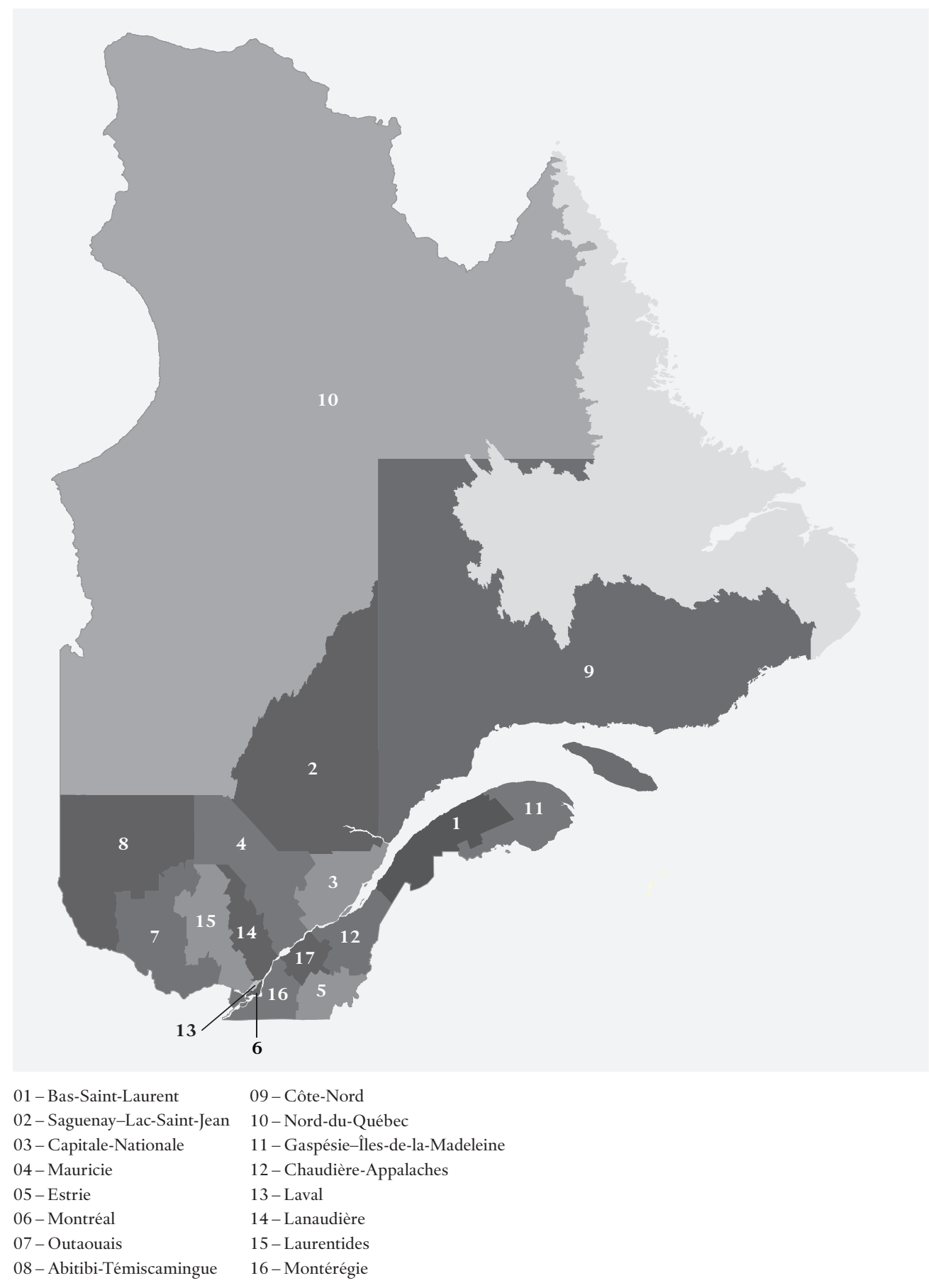

Source: Regroupement des associations forestières

régionales du Québec. Régions administratives du Québec.

$<$ http://www.afvsm.qc.ca/region.htm>

(consulté le 28 avril 2010). 
Du point de vue de l'urbaniste, une région est donc un territoire défini par le gouvernement où sont décentralisés certains pouvoirs et responsabilités.

\section{Du point de vue de l'historien}

Selon l'historien Chad Gaffield, la notion de région est liée à la question de spécificité. En effet, pour déterminer si un espace est une région, il importe de comprendre s'il possède une identité spécifique en posant certaines questions.

Cette identité permet-elle de distinguer cet espace des espaces limitrophes, et peut-être même d'autres régions? Les habitants de cette "région " et les observateurs de l'extérieur la perçoivent-ils comme une réalité? Existe-t-il, de la part des résidents de cet espace, une mentalité ou des conduites spécifiques qui nous permettraient de les différencier de ceux qui habitent ailleurs ${ }^{22}$ ?

La recherche de caractéristiques spécifiques, selon l'auteur, comporte ses risques car celles trouvées peuvent ne pas représenter l'ensemble d'un territoire (généralisation d'une spécificité locale) ou encore se retrouver ailleurs, dans d'autres régions. Il importe donc de s'intéresser aux grands traits d'un territoire et à leurs rapports mutuels.

\section{Du point de vue du géographe}

Selon Laurent Deshaies ${ }^{23}$, il est bien problématique de définir le terme "région ", car on observe une grande diversité d'opinions parmi les géographes eux-mêmes. Cet auteur rappelle qu'en général la région désigne un territoire d'échelle moyenne situé entre la nation et les espaces locaux. Après 1960, de grands changements se sont produits dans la définition du terme: "elle [la région] fut considérée par la suite comme un espace à construire pour soutenir l'intérêt de l'administration, de la décentralisation ou du développement régional $»^{24}$.

22 GAFFIELD, «La région: une combinaison spécifique d'éléments non spécifiques ", op.cit., p. 27-31.

23 DESHAIES, Laurent. "La notion de région en géographie». In. HARVEY, La région culturelle. Problématique interdisciplinaire, op. cit., p. 33-55.

24 Id., p. 36.
Des géographes considèrent que la région en tant que telle n'existe pas: «Selon eux, la région est avant tout un concept intellectuel qui sert à choisir certains traits de la réalité pour répondre à un besoin particulier ${ }^{25}$. " Le terme "région " ne peut, avancent-ils, posséder une définition absolue. C'est ce qui amène Deshaies à conclure que la meilleure définition est celle qui correspond le mieux aux objectifs poursuivis par un chercheur.

\section{Du point de vue du sociologue}

La notion de région en sociologie est issue des limites des recherches en géographie sur le sujet. Le point de vue du sociologue Bruno Jean rejoint en partie celui du géographe Laurent Deshaies, selon lequel la région ne peut être simplement tracée sur une carte à partir de critères géographiques.

La région n'est pas une réalité qui s'impose d'elle-même à partir d'une simple lecture de paysage, c'est d'abord une production sociale, c'est-à-dire une construction sociale, toujours inachevée, toujours en reconstruction, et où s'entremêlent autant un environnement et ses déterminismes que les aménagements volontaristes des collectivités. Car la région, c'est aussi une référence identitaire plus ou moins forte, qui donne un cadre spatio-temporel aux projets que font les communautés humaines pour aménager leur propre avenir ${ }^{26}$.

Jean insiste sur le fait que le développement régional doit être basé sur des approches visant la décentralisation des pouvoirs et tendre vers des processus endogènes. Bref, les stratégies et les méthodes de développement régional doivent converger vers ce qu'il nomme le "développement approprié».

Parler de l'autopromotion paysanne en

Afrique, de développement endogène, décentralisé ou local au Québec, c'est désigner une même réalité. Il s'agit de construire des projets de développement qui répondent

25 Id., p. 38.

26 JEAN, Bruno. "La région sous le regard sociologique:

la construction sociale du fait régional ". In. PROULX,

Marc-Urbain (dir.). Le phénomène régional au Québec.

Québec: Presses de l’Université du Québec, 1996, p. 134. 
à des besoins des communautés locales, à leurs attentes et qui s'inscrivent harmonieusement dans leur réalité socio-historique profonde ${ }^{27}$.

Pour la sociologue Andrée Fortin, les régions au Québec peuvent être classifiées selon différents types de "régions culturelles", ce qui peut aider à définir le terme. Dans une première catégorie, il y a Montréal et Québec, respectivement métropole et capitale nationale. Dans une deuxième catégorie il y a les régions périphériques à ces deux villes et par la suite il y a les régions excentriques dont fait partie la région du Saguenay-Lac-Saint-Jean. Comme le mentionne l'auteure, l'espace culturel d'un territoire se divise lui-même en régions (arts visuels, arts de la scène, architecture, etc.), chacune obéissant à des règles et à un réseau particulier. Définir la nature de la culture d'une région est donc très complexe. Fortin réussit à identifier quelques spécificités de la culture en région. Elle rejoint ainsi les nouvelles tendances en muséologie.

En fait, la culture en région a ses spécificités: celles-ci ne sont pas liées au contenu, à la forme mais à sa mise en ouvre, aux étapes de monstration et de légitimation. On observe à cette étape une véritable mobilisation des ressources communautaires $[\ldots]$ Il va sans dire que les institutions locales sont les partenaires essentiels de toute vie culturelle régionale ${ }^{28}$.

D'un point de vue sociologique, les spécificités des régions doivent donc être mises de l'avant et non perçues comme des inégalités que l'on doit abolir afin de promouvoir la diversité culturelle au sein d'une même nation.

\section{Du point de vue de l'ethnologue}

L'ethnologue conçoit la région comme étant un territoire où l'on observe des singularités sur le plan socioculturel. La définition de la notion

27 Id., p. 150

28 FORTIN, Andrée. "L'occupation culturelle de l'espace régional ". In. PROULX, Le phénomène régional au Québec, op. cit., p. 179. de région culturelle est donc implicite à la définition du concept de région. Tel que le résume Jocelyne Mathieu, ethnologue ${ }^{29}$, la région culturelle est en rapport avec l'environnement et l'adaptation d'une population à ce dernier. La culture régionale, quant à elle, est basée sur la perception qu'a la population d'elle-même et de l'Autre. Elle émet l'hypothèse que l'identité régionale est en bonne partie relative à la réalité économique d'un territoire, puisque la population s'adapte non seulement à son environnement naturel, mais également à son environnement économique. Une bonne perception de l'économie régionale engendre une meilleure perception de l'identité régionale. Du point de vue ethnologique, une région se définit donc par sa culture et sa culture est liée à la notion d'identité régionale de même qu'à une importante mobilisation des ressources communautaires.

\section{Du point de vue du gouvernement}

Dans son Bilan des portraits statistiques régionaux, Claude Edgar Dalphon résume quelques caractéristiques des régions excentriques (éloignées, selon l'auteur). Ce bilan, qui a été effectué pour le compte du MCCCf, est disponible sur son site Internet officiel sous l'onglet "Publications "; il nous porte à croire que les caractéristiques des régions excentriques suivantes sont celles reconnues par le gouvernement:

Les régions éloignées possèdent généralement les plus grands territoires et les plus petites populations du Québec. Elles sont aussi les seules à connaître une décroissance de leur population. Toutes les variables reliées à l'économie, dont le revenu personnel, y sont sous la moyenne québécoise, parfois par une forte marge. La situation est la même pour la scolarité, ces régions étant les seules dont la proportion de personnes possédant un diplôme postsecondaire se trouve sous la moyenne ${ }^{30}$.

29 MATHIEU, Jocelyne. «Le phénomène régional au Québec - Contribution ethnologique», In. PROULX, Le phénomène régional au Québec, op. cit., p. 157-170. 30 DALPHON, Claude Edgar. Bilan des portraits statistiques régionaux. <http://www.mcccf.gouv.qc.ca/fileadmin/ documents/publications/regions/bilan-portraits-statistiquesregionaux-2006.pdf> (consulté le 28 mars 2010), p. 13. 
Au niveau gouvernemental, le terme région désigne donc explicitement ${ }^{31}$ un échelon territorial et administratif situé entre l'espace national et l'espace local.

\section{La définition d'une région au Québec?}

Tous les auteurs cités précédemment affirment qu'une région ne peut être définie que par des critères géographiques et cartographiques. Une région est donc une production sociale toujours inachevée et en reconstruction, tout comme la société qui en occupe le territoire. Il apparait qu'elle est d'abord et avant tout un territoire spécifique que l'on distingue facilement des espaces limitrophes. Ce territoire est spécifique car il possède une identité qui l'est également.

À la lumière de ces informations, il s'avère plus prudent de définir un type de région au Québec: la région de type excentrique, dans le cas présent. Il est donc possible d'avancer que la région excentrique est caractérisée par un développement particulièrement endogène vu son grand éloignement des grands centres urbains. Ce développement endogène favorise une certaine autonomie culturelle qu'on observe plus difficilement dans les autres types de régions ${ }^{32}$ et qui est possible grâce à une plus grande mobilisation des ressources communautaires. De plus, ces territoires sont caractérisés par la présence d'une forte identité régionale, sont généralement constitués à $90 \%$ de population francophone ${ }^{33}$ et sont marqués par un taux de chômage supérieur à la moyenne québécoise.
31 Claude Edgar Dalphon, dans son bilan des portraits statistiques régionaux effectué pour le MCCCf, s'inspire des travaux de Fortin et Harvey pour construire sa propre typologie des régions au Québec. Il est donc possible de constater que, pour le gouvernement provincial, le Saguenay-Lac-SaintJean correspond à une région de type "éloigné».

32 HARVEY et FORTIN, «Production et diffusion culturelles en région...", op. cit., p. 13-34.

\section{L'interprétation de l'institution par ses caractéristiques intrinsèques}

Les musées en région au Québec ne peuvent être caractérisés par leur simple présence en région, puisque les différentes significations de ce terme ne font pas l'unanimité: il est possible d'établir une définition pour chaque type de région, mais pas pour l'ensemble de ces territoires. Les musées en région doivent donc être interprétés par leurs caractéristiques intrinsèques.

\section{La culture en région excentrique:} une vue d'ensemble de la situation Le Bilan Dalphon ${ }^{34}$ résume la situation de la culture en région excentrique. Selon ce bilan, ces territoires correspondent à ceux où l'on observe le plus faible taux de participation aux activités culturelles, sauf en ce qui a trait à la télévision et à la lecture des hebdomadaires régionaux. De plus, ce sont les régions où l'on trouve la plus faible quantité de main-d'œuvre spécialisée dans le secteur culturel, mais également les endroits où le taux d'équipements culturels par habitant est le plus élevé.

En 2003 et 2004, un comité d'évaluation national des institutions muséales (reconnues et soutenues - ou non - au fonctionnement par le ministère de la Culture) a produit, sous la présidence de Marie-Thérèse Bournival, un rapport ${ }^{35}$ rendant compte de la situation des musées québécois. L'information qu'il importe de retenir lorsqu'on s'intéresse à la question des musées en région est la suivante:

- Plus des deux tiers du soutien financier du

MCCCf sont alloués à quatre grands musées qui relèvent d'une loi spécifique.

- Le réseautage en région s'est amélioré entre 1999 et 2003. Certaines régions se sont par exemple dotées d'outils de promotion communs.

33 DALPHON, Bilan des portraits statistiques régionaux, op.cit.

34 Id.

35 BOURNIVAL, Marie-Thérèse. Des avancées remarquables. Avancées remarquables du réseau muséal du Québec en dépit de difficultés récurrentes et d'un contexte déstabilisant. Rapport de la présidence. Québec: Ministère de la Culture, des Communications et de la Condition féminine du Québec, janvier 2004. 
- Entre 1996-1997 et 2000-2001, les deux catégories d'organismes à avoir connu une baisse salariale sont les musées régionaux $(0,08 \%)$ et les lieux d'interprétation $(11,07 \%)$.

- L'ampleur des défis à relever et les salaires très bas empêchent la rétention du personnel muséal en région. Cela entraîne une grande perte d'expertise et un manque de stabilité dans les missions poursuivies.

- Des institutions régionales bien positionnées et mieux dotées en ressources partagent leur expertise avec d'autres institutions moins bien nanties.

\section{Des problèmes actuels}

\section{La distance entre le musée en région} et son milieu

Geneviève Provencher-St-Cyr, dans un article paru dans la revue Muséologies Les cahiers d'études supérieures d'avril $2008^{36}$, étudie le mouvement de distanciation entre les institutions muséales en région et leur communauté. Cette recherche s'appuie sur une étude théorique de l'évolution du rôle social du musée et sur une étude de cas au Musée de Charlevoix. Cette dernière a permis de mettre de l'avant une certaine dichotomie dans la perception qu'a la population de Charlevoix du musée régional, qu'elle perçoit comme "l'essence de Charlevoix mis en scène pour l'Autre ${ }^{37}$.

L'origine de cette distanciation se situe en général, selon l'auteure, au moment où le musée gagne de l'autonomie et se professionnalise: "l'institution devient alors un musée pour l'autre et cela, malgré sa mission de refléter le milieu et la provenance de sa collection ${ }^{38}$. Provencher-St-Cyr rappelle que les missions des musées en région, en général, gravitent autour de la notion d'identité et que ces institutions se doivent d'être un miroir qui reflète le plus fidèlement possible leur environnement.
Le milieu doit être inclus dans la structure du musée et le musée doit participer au développement du milieu.

\section{L'isolement des musées en région}

Provencher St-Cyr a développé un modèle d'action permettant de rapprocher les musées en région de leurs communautés. Philippe Dubé, muséologue, chercheur et enseignant, propose une solution à un autre problème du même type: celui de l'éloignement de ces musées des autres institutions et ressources culturelles sur le territoire régional.

Tel que l'observe et le résume Dubé ${ }^{39}$, les musées en région se trouvent isolés sur de très grands territoires. Cette situation engendre de nombreux problèmes qui ont des conséquences directes sur le fonctionnement de l'institution. Par exemple, les écoles sont souvent éloignées des musées et les coûts élevés de transport scolaire empêchent bien des classes d'y accéder. La recherche devient également plus complexe si les bibliothèques universitaires les plus proches sont à une distance considérable. Cette situation rend évidente la nécessité de repenser l'ingénierie culturelle de ces territoires afin de permettre aux musées de bien jouer leur rôle dans la société.

\section{Le manque de ressources humaines et financières}

Selon Dubé, les petits et les moyens musées (PMM) d'aujourd'hui ne sont plus en mesure de bien remplir leurs fonctions telles que décrites dans la définition du musée instaurée par l'International Council of Museum ${ }^{40}$. Par PMM, cet auteur fait référence à « une institution qui opère l'année durant avec un personnel permanent de trois à cinq individus maximum et ayant un budget annuel, en chiffres d'aujourd'hui [2001], de 175000 à 350000 dollars » ${ }^{41}$.

38 Id., p. 428.

39 DUBÉ, "À propos d'une certaine muséologie en région", op. cit. 40 DUBÉ, Philippe. «Le patrimoine territorial: ethnologie et muséologie». In. PICHETTE, Jean-Pierre. Entre Beauce et Acadie (dir.). Québec: Presses de l'Université Laval, 2001, p. 424.

41 Id. p. 428. coopérative. Une proposition d'ancrage des musées dan la vie régionale ". Muséologies Les cahiers d'études supérieures, vol. 2, $\mathrm{n}^{\circ}$ 2, 2008, p. 96-111.

37 DUBÉ, Philippe. «Le patrimoine territorial: ethnologie et muséologie ». In. PICHETTE, Jean-Pierre. Entre Beauce et Acadie (dir.). Québec: Presses de l'Université Laval, 2001, p. 424 . 
Tableau 2

Résultats de l'étude de terrain

\begin{tabular}{ll}
\hline $\begin{array}{l}\text { Réponses convergentes } \\
\text { Les musées en région au Québec: définition du concept et situation }\end{array}$ & $\begin{array}{l}\text { Pourcentage } \\
\text { de convergence }\end{array}$ \\
\hline $\begin{array}{l}\text { Un musée en région est un musée situé sur un territoire régional, } \\
\text { donc loin d'un grand centre urbain. }\end{array}$ & $50 \%$ \\
\hline $\begin{array}{l}\text { Un musée en région correspond à [énumération de problèmes } \\
\text { rencontrés par ces institutions selon le professionnel rencontré]. }\end{array}$ & $50 \%$ \\
\hline $\begin{array}{l}\text { Les musées dans les grands centres urbains sont plus gros } \\
\text { et plus compartimentés. }\end{array}$ & $50 \%$ \\
\hline $\begin{array}{l}\text { Le musée régional doit assurer un rôle de leader, de partenaire } \\
\text { et de référence au sein d'un réseau d'institutions muséales régionales. }\end{array}$ \\
$\begin{array}{l}\text { Cette institution a le mandat de collectionner, de protéger et de } \\
\text { diffuser le patrimoine de l'ensemble d'une région. }\end{array}$ \\
$\begin{array}{l}\text { Un musée en région, au contraire d'un musée régional, représente } \\
\text { une portion d'un territoire et donc d'une population. }\end{array}$ \\
$\begin{array}{l}\text { Les musées en région sont aux prises avec de grands problèmes } \\
\text { financiers, les coûts de production y étant définitivement plus élevés } \\
\text { que dans les grands centres urbains. }\end{array}$ \\
$\begin{array}{l}\text { Les musées en région sont aux prises avec de lourds problèmes } \\
\text { de ressources humaines, la rétention de celle-ci étant complexe } \\
\text { vu les piètres conditions salariales offertes par les institutions. }\end{array}$ \\
$\begin{array}{l}\text { Le rôle social du musée en région est fondamental. L'institution } \\
\text { existe d'abord pour la population locale et doit être au cœur } \\
\text { de celle-ci. }\end{array}$ \\
$\begin{array}{l}\text { Ce que la population et les visiteurs pensent réellement du } \\
\text { musée est déduit par les directeurs (revue de presse, observation } \\
\text { des visiteurs en salle ou autre) et aucune méthode ne permet } \\
\text { de réellement savoir ce que les visiteurs pensent du musée } \\
\text { et de son contenu. }\end{array}$ \\
\hline
\end{tabular}


Le manque de ressources financières et humaines ne leur permet pas de faire, par exemple, une quantité significative de recherches ou de conserver leurs collections selon les normes établies. Selon Dubé, cette crise de fonctionnement peut être résolue en revoyant les fonctions même du musée et en les adaptant à la réalité des petits et moyens musées.

\section{Un musée en région au Québec:} ses caractéristiques intrinsèques

Le musée en région au Québec est une institution qui voit son développement intimement lié aux notions de coopération et d'inclusion, car la culture en région s'épanouit avec le concours des ressources communautaires. Les problèmes singuliers que ce musée doit surmonter concernent le manque de main-d'œuvre qualifiée et l'isolement sur un grand territoire dont elles constituent parfois le seul lieu où l'on diffuse de la culture. D'autres problèmes vécus par les musées en région correspondent la plupart du temps au modèle du "petit et moyen musée $»^{42}$ : le musée en région centre la plupart du temps sa mission sur la définition, la protection et la diffusion de l'identité régionale.

\section{L'analyse des données récoltées sur le terrain}

L'étude de terrain est primordiale dans le cadre de cette recherche, car elle permet de nuancer les informations trouvées dans la littérature. Le territoire québécois étant très vaste, il n'a évidemment pas été possible d'étudier toutes les régions administratives. En ne ciblant qu'une région sur douze comme terrain d'enquête, cette étude de cas constitue un outil qui doit mener à une généralisation et peut être qualifiée d'instrumentale ${ }^{43}$. Des entretiens non directifs ${ }^{44}$ avec les directeurs de quatre musées, réalisés dans la région du Saguenay-Lac-SaintJean, ont permis d'obtenir une plus grande quantité d'information à caractère qualitatif ${ }^{45}$ en explorant la pensée des professionnels sur certains sujet.

$42 I d$.

43 STAKE, Robert E. "Case Studies ». In DENZI,

Norman K. et Yvonna S. LINCOLN. Handbook of Qualitative Research. Californie: Sage Publications, 2000, p. 437. 44 FERRÉOL, Gilles et Philippe DEUBEL. Méthodologie
Le tableau 2 résume les résultats de l'étude de terrain. Il est important de noter que le pourcentage de convergence des données n'indique pas le nombre de directeurs qui ont appuyé l'énoncé, mais bien le nombre de directeurs qui ont naturellement mentionné l'énoncé dans leurs réponses.

\section{Synthèse des résultats des entretiens}

Lors des rencontres avec les directeurs, la question concernant la définition d'un "musée en région au Québec "s'est avérée problématique pour chacun. Il est donc facile de comprendre que, sur le terrain, la définition de «musée en région" demeure tout autant nébuleuse que dans la littérature. On définit cette institution par rapport à son lien avec le territoire, par rapport aux problèmes particuliers qui empêchent son développement ou encore en la comparant avec une institution située dans un grand centre urbain.

Les propos des directeurs et la littérature abondent dans le même sens: les problèmes de financement et de ressources humaines sont intimement liés à la définition du concept de musée en région. Toutefois, la totalité des professionnels rencontrés s'entendent pour affirmer que ce problème de financement n'est pas seulement attribuable au fait que les subventions gouvernementales accordées sont largement insuffisantes. Ce problème est également dû au fait que les coûts de production sont réellement plus élevés en région (par exemple, le matériel nécessaire pour la conception des vitrines est vendu à Montréal). Cela n'est pas abordé dans la littérature: les entretiens liés à cette recherche permettent donc d'apporter une précision au problème de sous-financement de ces institutions.

La revue de la littérature permet de constater que le rôle social du musée en région est fondamental et doit être mis de l'avant dans 
une optique de développement culturel régional. Les entretiens réalisés démontrent que les muséologues du Saguenay-Lac-Saint-Jean abondent aussi dans ce sens. Toutefois ces derniers avouent ne pas posséder de méthode d'analyse des clientèles qui permettrait de mieux connaître les besoins et les attentes de la population locale en matière de culture. Ce problème lié à l'étude des publics en région n'est pas couvert par la littérature alors qu'il est un enjeu majeur. C'est précisément ce que la présente enquête a, entre autres, permis de relever.

\section{Qu'est-ce qu'un musée en région excentrique au Québec?}

À la lecture de quelques articles traitant du sujet des musées en région au Québec, il apparait que ces institutions vivent des difficultés communes. Certaines d'entre elles, comme le sous-financement et la distance qui sépare souvent ces institutions de leur milieu, sont des difficultés qui peuvent être éprouvées par des musées de tout type partout au Québec. Toutefois, en considérant l'ensemble des données recueillies dans le cadre de cette recherche, il apparaît que ces écueils sont de plus grande ampleur en région en raison de la faible densité de population de ces territoires et la distance qui sépare ces musées des grands centres urbains et de leurs services. D'autres problèmes comme le manque de ressources humaines et l'isolement physique des institutions font aussi partie des caractéristiques intrinsèques des musées en région au Québec.

La définition d'une région excentrique au Québec, l'identification de problèmes communs aux institutions situées sur ces territoire et des entretiens avec les directeurs de ces institutions nous permettent de proposer une réponse à la question: qu'est-ce qu'un musée en région excentrique au Québec?
Le musée en région excentrique au Québec est une institution qui voit son développement intimement lié aux notions de coopération et d'inclusion de la population, qui est aux prises avec un problème majeur de manque de main-d'œuvre qualifiée et qui doit gérer son isolement sur un grand territoire dont elle constitue parfois le seul lieu où l'on diffuse de la culture. De plus, le musée en région excentrique centre la plupart du temps sa mission sur la définition, la protection et la diffusion de l'identité régionale.

\section{Conclusion}

Le sujet des musées en région au Québec est très peu présent dans la littérature scientifique en muséologie. Leurs caractéristiques, leur mode de fonctionnement et les défis auxquels ils doivent faire face sont méconnus. Cela est fort probablement attribuable au fait que la notion de «région " elle-même n'est pas encore réellement définie, ni stabilisée. Pour le moment, apparaît l'impossibilité de définir ce qu'est un musée en région au Québec. Toutefois, ceux situés en région excentrique pourraient être définis et éventuellement reconnus comme des institutions muséales d'un autre type.

Étant donné toutes les particularités propres à chaque région du Québec, la généralisation des résultats d'une recherche ayant pour terrain une seule d'entre elles demeure risquée et doit être faite avec prudence. Comme l'estiment Andrée Fortin et Fernand Harvey ${ }^{46}$, les autres types de régions possèdent des caractéristiques intrinsèques différentes qui influent sur leur ingénierie culturelle et donc sur le fonctionnement de leurs institutions muséales. Les résultats de la présente recherche pourraient être applicables à d'autres régions excentriques étant donné que ces territoires possèdent plusieurs caractéristiques communes. Toutefois,

46 HARVEY et FORTIN, "Production et diffusion culturelles en région...", op. cit. 
une étude comparative entre les régions de ce type serait nécessaire afin de déterminer jusqu'où la vie culturelle peut y être similaire.

L'amélioration de la situation des musées en région constitue un enjeu majeur pour les générations futures. Ces institutions demeurent souvent les seules gardiennes des cultures régionales au Québec et constituent par le fait même d'importants acteurs dans la définition, la conservation et la diffusion de l'identité de la nation québécoise. Dans un tel contexte, les propos du sociologue Bruno Jean prennent une signification encore plus importante:

Le maintien de la biodiversité est certainement l'une des grandes luttes du mouvement environnementaliste dont la justification n'est plus à faire. Pourtant, au même moment, le nivellement socio-économique et culturel des cultures régionales de par le monde est certainement un phénomène qui inquiète trop peu les milieux scientifiques de même que les médias et l'opinion publique ${ }^{47}$.

47 JEAN, "La région sous le regard sociologique...", 
Meggie Savard, Translated by Allana Carlyle

\section{Quebec's Regional Museums}

Quebec society as well as the organization of its territory has much evolved over the course of recent decades. Since the second half of the $19^{\text {th }}$ century, vast wooded landscapes have been cleared and colonized, forming new administrative regions.

These territories, often far removed from urban centres, develop differently and must overcome difficulties that the populations of Quebec and Montreal do not have to face: rural depopulation of young people or low school attendance rate. These same territories are supported by diverse government grants, and yet, their cultural institutions do not receive any specific support despite the additional challenges they must face.

The regional museum is not recognized by different levels of government or by the International Counsil of Museums (ICOM). Is it possible to create a definition for the regional museum in Quebec? Can we envision the creation of a new type of museal institution so that these museums can benefit from government support adapted to their individual needs? With the goal of answering these questions, this article will propose a definition for the regional museum in Quebec based on a multidisciplinary literature review and case study from Saguenay-Lac-Saint-Jean.

Literature shows that it is difficult to define a Quebec region. Opinions on this topic vary from one discipline to another. For the purpose of this analysis, peripheral, outlying regions will be targeted. These regions possess different characteristics allowing them to be easily differentiated from other types of territories, facilitating the study of their institutions. It is recognized that that outlying regions are characterized by endogenous development, a certain cultural autonomy, the presence of a strong regional identity, a population comprised of a $90 \%$ francophone majority as well as a welfare rate higher than the Quebec average.

The results of the case study corroborate the results of the literature review. Furthermore, they shine a light on the gravity of the financial problems and lack of human resources as well as the lack of tools allowing for the analysis of the clientele.

Given that different types of regions exist, it is imprudent to define the regional museum based only on its local in the provincial territory. These museal institutions could be equally defined based on the reoccurring problems that arise.

It is possible to suggest that the regional museum in Quebec is an institution whose development is intimately linked to the notions of cooperation and inclusion, as the culture of this territory blossoms with the support of community resources. These museums must overcome unique challenges, including a lack of qualified workforce and isolation 
in a large territory where they are often the only site diffusing culture. This museal institution most often focuses its mission on the definition, protection and diffusion of regional identity. 